MS07-04

\section{Crystal structure of TraN, a regulator of conjugative DNA-transfer, in complex with its cognate DNA binding site}

Walter Keller ${ }^{1}$, Verena Kohler ${ }^{1}$, Nikolaus Goessweiner-Mohr ${ }^{1}$, Andreas Aufschnaiter ${ }^{1}$, Christian Fercher ${ }^{2}$, Ines Probst ${ }^{3}$, Tea PavkovKeller $^{1}$, Kristin Hunger ${ }^{1}$, Heimo Wolinskiv ${ }^{1}$

1. Institute of Molecular Biosciences, University of Graz, Graz, Austria

2. Australian Institute for Bioengineering and Nanotechnology, The University of Queensland, Brisbane, Australia

3. Division of Infectious Diseases, University Medical Center Freiburg, Freiburg, Germany

email: walter.keller@uni-graz.at

Bacterial conjugation is the most prevalent route of DNA transfer between bacteria and mediates the rapid spread of bacterial resistances within bacterial communities. Type IV secretion system (T4SS), elaborate molecular machines, are responsible for the efficient transport of nicked, single-stranded plasmid DNA across the cell walls of the donor as well as the recipient cell [1]. We investigate the T4SS from the antibiotic resistance plasmid pIP501, which is encoded within a single operon comprised of 15 putative transfer factors. The $14^{\text {th }}$ open reading frame encodes TraN, a specific double-strand DNA binding protein, which recognizes a $34 \mathrm{bp}$ long stretch of DNA positioned $72 \mathrm{bp}$ upstream of the oriT nick site [2]. The structure of unbound TraN has been determined previously [2]. Here we present the TraN-DNA complex structure determined at $1.93 \AA$ and show that TraN acts as a repressor of the pIP501 encoded T4SS. The repressor function of TraN has been shown in in vivo experiments: an $E$. faecalis traN deletion strain exhibits highly increased transfer rates, as well as elevated mRNA and protein levels of the all tested tra-operon components, including the putative T4SS complex member TraM. Based on the detailed analyses of the TraN-DNA interaction we identified a further high-affinity binding site within the tra-operon (upstream of the traN gene). Furthermore, we show that related plasmids of the Inc 18 family that encode a TraN homolog also harbor at least one conserved TraN binding site. We therefore conclude that the proposed regulatory role of TraN is conserved in the related plasmids.

References:

[1] Alvarez-Martinez CE, and Christie PJ (2009) Microbiol Mol Biol Rev 73, 775-808.

[2] Goessweiner-Mohr N, Eder M, Hofer G, Fercher C, Arends K, Birner-Gruenberger R, Grohmann E and Keller W (2014) . Acta Cryst D70, 2376-2389.

Keywords: Type 4 Secretion Systems, Repressor, antibiotic resistance
MS07-05

\section{RNA specificity of NHL domains revisited: LIN-41/TRIM71 binds a defined RNA stem-loop element via shape and electrostatic complementarity}

Heinz Gut ${ }^{1}$, Pooja Kumari ${ }^{1}$, Florian Aeschimann ${ }^{1}$, Dimos Gaidatzis ${ }^{1}$, Jeremy J. Keusch ${ }^{1}$, Anca Neagu' ${ }^{1}$, Katarzyna Pachulska-Wieczorek ${ }^{2}$, Helge Grosshans ${ }^{1}$, Rafal Ciosk ${ }^{1}$

1. Friedrich Miescher Institute for Biomedical Research, Basel, Switzerland

2. Institute of Bioorganic Chemistry, Polish Academy of Sciences, Poznan, Poland

email: heinz.gut@fmi.ch

RNA-binding proteins regulate a myriad of biological pathways by tightly controlling the fate of ribonucleic acids. For this purpose, nature has evolved an arsenal of different RNA binding domains specialized to specifically associate with a variety of target RNAs. While some of these protein domains show preference for a defined RNA sequence motif, others interact primarily with structured RNA elements. For both cases, examples for recognition of single-stranded or double-stranded RNA stretches have been identified. Only very recently, the NHL domain of TRIM-NHL protein family members, which include some of the key regulators of cell proliferation and differentiation, has been added to the repertoire of RNA-binding folds. The TRIM-NHL family member Brat from Drosophila melanogaster has been shown to specifically bind, via its NHL domain, a short single-stranded RNA stretch containing a defined sequence motif [1]. However, it remains largely unexplored how the many other family members featuring the WD40-like beta-propeller of the NHL domain interact with RNA. Here we present a completely different solution for recognition of an RNA element by the NHL domain of another TRIM-NHL family member. The Caenorhabditis elegans LIN-41 protein, a well-studied target of the let-7 miRNA, regulates developmental processes in the soma and has been shown to reprogram germ cells into pluripotency during the oocyte to embryo transition. Similarly, its human homolog TRIM71 plays a role in cellular plasticity by regulating the transition from differentiated to pluripotent cells. In both worms and mammalian cells, LIN-41/TRIM71 function has been linked to degradation or translational repression of specific mRNAs, but how these RNA molecules are recognized has remained a mystery [2]. By combining computational analysis, X-ray crystallography, and in vivo studies in worms and human cells, we find that the NHL domain of LIN-41/TRIM71 binds a short stem-loop RNA element, which is present in multiple copies in the 5'- or 3'-UTRs of well-established LIN-41/TRIM71 mRNA targets. Based on the computational and structural data, we suggest a LIN-41 RNA response element of varying sequences capable of folding into the correct 3D RNA-hairpin structure to achieve specificity by shape and electrostatic complementarity to a positively charged, shallow pocket in the NHL domain of LIN-41/TRIM71 [3].

References:

[1] Loedige, I. et al. (2015). Cell Rep. 13(6), 1206-1220.

[2] Aeschimann, F. et al. (2017). Mol Cell, 65(3), 476-489.

[3] Kumari, P. et al. (2018). Nat Commun. 9(1), 1549. 